\title{
Ação do cariostático nas lesões de cárie durante a COVID-19
}

\author{
Cariostatic action in the caries lesions during COVID-19
}

Recebido: 26/09/2021 | Revisado: 03/10/2021 | Aceito: 05/10/2021 | Publicado: 12/10/2021

\author{
Vitória Moura \\ Universidade Brasil, Brasil \\ E-mail: vitoriasmouraa@gmail.com \\ Jennifer Lima \\ Universidade Brasil, Brasil \\ E-mail: jenn.1102@hotmail.com \\ Caleb Shitsuka \\ Universidade Brasil, Brasil \\ E-mail: cashitsuka@gmail.com
}

\begin{abstract}
Resumo
Objetivo: Apresentar o uso do diamino fluoreto de prata (DFP) utilizado no controle da cárie dentária na infância, em tempos de pandemia pela COVID- 19. Métodos: A busca pelos artigos para a revisão de literatura foi executada na base de dados da Scielo, PubMED e Google Scholar. Resultados: Diante da pandemia da COVID- 19, os cirurgiões dentistas tiveram um grande desafio em um cenário passível de contaminação e o uso do DFP ganhou um espaço diante desse desafio, considerado um tratamento de mínima intervenção, um material eficaz, de baixo custo e de fácil aplicação e que dispensa o uso de instrumentos rotatórios, foi utilizado para paralisar as lesões de cárie e para a prevenção de novas lesões. Conclusão: O diamino fluoreto de prata utilizado para paralisar e prevenir lesões de cárie, é um material eficaz, de baixo custo, fácil aplicação e que diminui a contaminação diante da COVID- 19.
\end{abstract}

Palavras-chave: Cárie dentária; Diamino fluoreto de prata; COVID-19.

\begin{abstract}
Objective: Present the use of silver diamine fluoride (SDF) used in the control of dental caries in childhood, in times of pandemic by COVID-19. Methods: The search for articles for the literature review was performed in the database of Scielo, PubMED and Google Scholar. Results: In view of the COVID-19 pandemic, dental surgeons had a great challenge in a scenario susceptible to contamination and the use of SDF gained space in the face of this challenge, considered a minimal intervention treatment, an effective, low-cost and Easy to apply and does not require the use of rotating instruments, was used to paralyze caries lesions and to prevent new lesions. Conclusion: The silver diamine fluoride used to paralyze and prevent caries is an effective material, of low cost, easy to apply and decreases the risk of contamination to COVID-19.
\end{abstract}

Keywords: Dental caries; Cariostatic; COVID-19.

\section{Introdução}

Em tempos atuais, o assunto predominante no mundo é a pandemia da doença do coronavírus, (COVID 19) causada por um vírus da família Coronaviridae da ordem Nidovirales, descoberto em 2019, em Wuhan, na China. Desde então, o vírus vem se propagando rapidamente no mundo. Sua transmissão se dá por meio da saliva e de secreções da nasofaringe de pessoas infectadas, suas vias de transmissão são através do contato direto com superfícies contaminadas ou através de aerossóis. (Lana et al., 2020)

Os profissionais de saúde constituem um grupo de risco para a COVID-19, por estarem expostos diretamente aos pacientes infectados. Com o avanço da pandemia esses profissionais tiveram de adotar medidas de proteção e prevenção contra essa infecção, visando reduzir ou eliminar a produção dos aerossóis no uso de instrumentos rotatórios no consultório por conta da contaminação maior do vírus durante os atendimentos, o que traz maiores riscos de contaminação cruzada. (Meng et al., 2020)

Deste modo, a Academia Americana de Odontopediatria, (Academia Europeia de Odontopediatria) e Nacional (Academia Brasileira de Odontopediatria) restringiram a indicação de procedimentos clínicos odontopediátricos para casos de 
urgência. Embora seja relatado que o número de casos em crianças é baixo em comparação com os adultos, estas também são propensas à infecção pela COVID-19 (Sousa et al., 2021; Shitsuka et al., 2019)

Na primeira infância a doença cárie é a mais prevalente das doenças que ocorrem nessa fase e na maioria das vezes não são tratadas, o que pode ter profundo impacto na vida das crianças. Sua etiologia é multifatorial. Desenvolve-se a partir da presença do biofilme dental, que é o responsável por mediar a desmineralização dos tecidos dentários denominados como esmalte e dentina. Para sua ocorrência, há a necessidade da interação de três fatores: microorganismos cariogênicos (Streptococcus mutans), substrato fermentável (como a sacarose) e um hospedeiro vulnerável. (Losso et al., 2009)

Diante de uma pandemia, para evitar a disseminação do vírus e reduzir o risco de contaminação entre pacientes, os profissionais passam a priorizar a realização de procedimentos odontológicos minimamente invasivos como o cariostático (diamino fluoreto de prata). Um dos agentes que paralisa e previne a doença cárie sem o uso de instrumentos rotatórios, com um tempo menor de trabalho, de fácil aplicação, custo acessível e que tem se mostrado eficaz no controle da doença cárie. (Dias et al., 2021)

O objetivo deste estudo é, por meio da literatura, apresentar uma medida preventiva que paralisa e previne a doença cárie, sem a produção de aerossóis, sem grandes riscos de contaminação e com um tempo menor de trabalho no consultório odontológico.

\section{Metodologia}

Foi realizada uma revisão de literatura (Estrela, 2018) com busca nas bases de dados: Scielo, PUBMED, Google Scholar. Foram selecionados 36, nos idiomas: inglês, português com recorte temporal 2007 de 2021.

\section{Resultados e Discussão} COVID-19

O SARS-CoV-2 é um vírus identificado como a causa de um surto da doença COVID-19, detectado pela primeira vez em Wuhan - China em dezembro de 2019. Desde o início dos casos, a Organização Mundial de Saúde (OMS) esteve acompanhando a evolução da doença e em 11/03/2020, foi declarado o estado de pandemia de COVID-19. Esse termo é usado para descrever uma situação infecciosa que ameaça muitas pessoas ao redor do mundo simultaneamente. (Pinto et al., 2020; Anvisa et al., 2021)

A COVID-19 é uma doença infecciosa causada pelo novo coronavírus (SARS-CoV-2) e tem como principais sintomas febre, cansaço e tosse seca. Alguns pacientes podem apresentar dores, congestão nasal, dor de cabeça, conjuntivite, dor de garganta, diarreia, perda de paladar ou olfato, erupção cutânea na pele ou descoloração dos dedos das mãos ou dos pés. Esses sintomas geralmente são leves e começam gradualmente. Algumas pessoas são infectadas, mas apresentam apenas sintomas muito leves. (OPAS et al., 2021; Guinancio et al., 2020)

A maioria das pessoas (cerca de 80\%) se recupera da doença sem precisar de tratamento hospitalar. Uma em cada seis pessoas infectadas por COVID-19 fica gravemente doente e desenvolve dificuldade de respirar. As pessoas idosas e as que têm outras condições de saúde como pressão alta, problemas cardíacos e do pulmão, diabetes ou câncer, têm maior risco de ficarem gravemente doentes. No entanto, qualquer pessoa pode pegar a COVID-19 e ficar gravemente doente. (OPAS et al., 2021)

Os sintomas de COVID-19 grave incluem: dispnéia (dificuldade respiratória), perda de apetite, confusão, dor ou aperto no peito persistente, alta temperatura (acima de $38^{\circ} \mathrm{C}$ ). Outros sintomas menos frequentes: Irritabilidade, perda de consciência (às vezes associada a convulsões), ansiedade, depressão, transtornos do sono, complicações neurológicas mais 
sérias e raras, como acidentes vasculares cerebrais, edema cerebral, estado delirante e lesões neurais. (Moraes et al., 2020; OMS et al., 2021)

Cerca de $80 \%$ dos casos são leves e se recuperam da doença sem a necessidade de tratamento especial. No entanto, em $15 \%$ dos casos são classificados como gravemente enfermos e os 5\% restantes são categorizados como gravemente enfermos. Em casos graves e críticos, a doença respiratória aguda pode causar pneumonia, insuficiência renal e até a morte. (Alharbi et al., 2020)

\section{Cárie Dentária}

A cárie dentária é uma doença transmissível e complexa do biofilme que cria períodos prolongados de baixo pH na boca, resultando na perda líquida de minerais dos dentes. Historicamente, o modelo de doença para cárie dentária consiste em streptococcus mutans e Lactobacillusespécies. (Kutsch et al., 2013)

O termo Cárie Dentária é utilizado para caracterizar lesões em diferentes estágios de desenvolvimento. Estes processos patológicos ocorrem nas superfícies dos dentes por meses ou até anos. (Batista et al., 2020). Seu diagnóstico nas fases iniciais é de extrema importância e deve ser tratada de forma adequada, para que não avance até à cavitação em dentina e/ou necrose da polpa dentária e afete a qualidade de vida do paciente. (Santana et al., 2018)

O Ministério da Saúde brasileiro relatou que a doença afeta mais de 50\% das crianças de até 5 anos, aproximadamente 80\% dos adolescentes e quase 100\% da população adulta (Brasil et al., 2011). Os índices de cárie entre adolescentes são mais elevados do que na infância, sendo expressivo o aumento da doença num período crítico de transição para a fase adulta. Por isso, identificar os determinantes sociais e individuais da cárie dentária entre os adolescentes pode contribuir para a prevenção da cárie e para a promoção da saúde bucal. (Frias et al., 2007)

As características epidemiológicas e socioeconômicas da população, suas condiç̃̃es, hábitos e estilos de vida, entre outros fatores, definirão as ações a serem adotadas para promoção, proteção e recuperação da saúde, para evitar estratégias equivocadas, que não são adequadas ao local. (Santana et al., 2018)

Quando a presença de lesão cariosa cavitada em dentina torna-se um fator retentivo para biofilme, é necessária uma intervenção mais invasiva para remover o tecido cariado e selar a cavidade. Nesse caso, pode ser implementado o Tratamento Restaurador Atraumático. (Santana et al., 2018; Maltarollo et al., 2020). Essa técnica preconiza a remoção do tecido cariado com instrumentos de corte manual, dispensando anestesia, isolamento absoluto e instrumentos rotatórios, o que permite sua aplicação em locais sem consultório odontológico. (Kikwilu et al., 2009)

\section{Tratamento Restaurador Atraumático}

O Tratamento Restaurador Atraumático surgiu na Tanzânia na década de 80, com o intuito de realizar um tratamento odontológico mais acessível, pois existiam regiões que a situação era muito precária, como por exemplo a falta de energia, dificultando a utilização dos motores odontológicos, sendo substituídos por escavadores manuais na remoção dos tecidos cariados onde posteriormente a cavidade seria preenchida com cimento de policarboxilato. (Navarro et al., 2015; Coelho et al., 2020)

O ART foi apresentado pela primeira vez aos profissionais brasileiros numa conferência, durante o 5th World Congress on Preventive Dentistry, realizado na cidade de São Paulo, em 1995, ou seja, um ano após ser reconhecido pela Organização Mundial da Saúde. (Massara MLA et al., 2010). A OMS descreveu o Tratamento Restaurador Atraumático como um novo procedimento revolucionário para tratar a cárie dentária, com grande potencial de melhorar a saúde bucal de muitos cidadãos, em diferentes partes do mundo, os quais não tinham acesso a cuidados bucais. (Leal Sc et al., 2012) 
A técnica do ART é constituída por um conjunto de medidas preventivas tais como a fluorterapia, orientação de higiene e dieta e uso de selantes, que já era utilizada no Brasil, porém a abordagem de lesões em dentina foi o que criou maior impacto diante da técnica (Massara et al., 2012, Risemberg et al., 2021; Pinheiro et al., 2021). Deve ressaltar que o Tratamento Restaurador Atraumático deve ser precedido de uma educação em saúde para controle e prevenção dos fatores etiológicos da doença cárie, esse controle deve ser realizado e monitorado através de consultas. (Garbin et al., 2008)

Com a evolução e desenvolvimento da técnica o uso do Tratamento Restaurador Atraumático está sendo introduzido cada vez mais na Odontologia moderna por ser incluído na Técnica de Mínima Intervenção, o ART não está sendo utilizado somente em países de baixa renda, está presente em consultórios particulares e o conceito está incluído no currículo acadêmico de muitas universidades de Odontologia de diversos países. (Frenchen et al., 2012; Coelho et al., 2020)

O ART é um tratamento muito eficiente pois ele não necessita de equipamentos odontológicos, o Cirurgião Dentista e sua equipe conseguem atuar em áreas de sua responsabilidade que estão distantes da sua unidade de saúde, incluindo centros comunitários, escolas e igrejas; O paciente se mantém tranquilo durante o procedimento, pois ele é indolor. Devido à ausência de dor, o paciente fica mais tranquilo para absorver as instruções e técnicas motivacionais de educação para a saúde. Por ser uma técnica simplificada e rápida, geralmente a equipe consegue restaurar vários elementos na mesma consulta, reduzindo o número de retornos até a alta do paciente, com isto a ausência ao trabalho e escola não será mais um empecilho para o paciente frequentar o consultório e conseguir finalizar o tratamento odontológico. (Monnerat et al., 2013).

\section{Diamino Fluoreto de Prata}

O DFP é um sal (Ag(NH3)2F) que contém em sua formulação íons prata e o fluoreto que formam um complexo com a amônia. Atualmente, não existe um consenso a respeito do seu mecanismo de ação na dinâmica multifatorial do processo de cárie; contudo, tem sido sugerido que o DFP promove: aumento da remineralização e diminuição da desmineralização pela ação do fluoreto na formação de fluorapatita e fluoreto de cálcio, preservação do colágeno presente na dentina por meio da inibição das metaloproteinases e redução do crescimento e a adesão de bactérias pela ação da prata. (Zhao et al., 2018).

Esse material possui três mecanismos de ação, sendo o primeiro o da obstrução dos túbulos dentinários. O segundo mecanismo é a ação cariostática, que se dá por meio do produto da reação entre o DFP e o componente mineral do dente que aumenta a resistência à descalcificação ácida. O terceiro mecanismo é a ação enzimática, que se caracteriza pelo aumento da resistência da proteína dentinária. (Santos et al., 2012)

Os estudos mostram que o diamino fluoreto de prata (DFP) é um agente fluoretado, seguro e não oneroso, que vem se mostrando efetivo no controle da cárie radicular, com melhor custo-benefício. Sua ação efetiva em dentina é baseada na remineralização da fase inorgânica e diminuição da degradação da fase orgânica, com recuperação de toda a complexidade estrutural dentinária. Efeitos adversos relatados do DFP estão relacionados principalmente ao escurecimento da superfície após a sua aplicação. (Dias et al., 2021)

Também conhecido como solução cariostática, o DFP apresenta em sua composição hidróxido de amônia, nitrato de prata, hidróxido de cálcio, ácido fluorídrico e solvente. No Brasil são comercializados nas concentrações de 10, 12, 30 e 38\%,

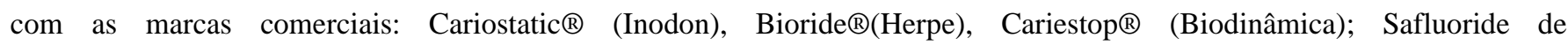
Walter®(Polidental), Cariestop® (Biodinâmica); Saforide®. (Wambier et al., 1995)

São várias as indicações clínicas do diamino fluoreto de prata: redução da sensibilidade da dentina, desinfecção de canal radicular, detecção de cáries incipientes, endurecimento da dentina amolecida para facilitar o preparo cavitário e protético, prevenção de cáries recorrentes e promoção do aumento da força de união do cimento fosfato de zinco à superfície dentária. Mas, entre todas as indicações mencionadas, a mais importante é a aplicação do diamino fluoreto de prata, com o objetivo de paralisar as lesões de cárie agudas em crianças, principalmente nos primeiros anos de vida. (Ando T) 
Algumas propriedades cariostáticas do DFP são o aumento da resistência do esmalte, inibição da formação de placa, diminuição da produção ácida dos micro-organismos da dentina cariada, redução da população dos Streptococcus mutans e obliteração dos canalículos dentinários expostos. (Ando et al., 1981)

Apesar de todas suas qualidades e vantagens, um dos desafios desse produto é seu indesejável efeito antiestético, dependendo da superfície na qual é aplicado. Como um dos componentes deste medicamento é a prata, provoca manchas de tom acastanhado nos dentes nos quais é aplicado. No caso de contato acidental da substância com pele ou mucosa, o manchamento também pode ocorrer. Essas manchas são reversíveis e desaparecem aproximadamente após uma semana. (Bueno et a., 2020)

Antes da aplicação do diâmino fluoreto de prata, deve-se informar os responsáveis que na região em que o processo da cárie esteja presente, ocorrerá o escurecimento e caso ocorra contato desta substância com tecido mole, por exemplo, a gengiva, formando uma área esbranquiçada. (Guedes et al., 1999)

A usabilidade do DFP em tempos de pandemia deve ser transmitida nas aulas remotas e presenciais dos cursos de Odontologia para que sejam aplicadas na prática profissional e o cirurgião dentista tenha o conhecimento utilizando esse produto que tem importantes propriedades durante o atendimento odontológico. (Valente et al., 2021; Santo, 2016; Rocha et al., 2021)

\section{Considerações Finais}

O diamino fluoreto de prata é uma ótima opção de tratamento minimamente invasivo, considerado eficaz, de fácil aplicação e de custo acessível para o controle e prevenção das lesões de cárie dentária na infância, principalmente em um cenário desafiador que estamos vivendo atualmente.

\section{Agradecimentos}

Agradecemos a Deus, ao senhor Antônio Moura, a senhora Rosângela Lima, ao senhor Evandro Lima e aos professores da Universidade Brasil.

\section{Referências}

Alharbi, A., Alharbi S., \& Alqaidi, S. (2020). Guidelines for dental care provision during the covid-19 pandemic. Saudi dent J. 2020;32(4),181-6.

Antunes, C.M.T.B., Luciano, C.C., Bahia, J.C., Bastos, R.M.A.F.P., \& Ando, T. (1981) Indicações do uso do Diaminofluoreto de prata (Sa-foride) em odontopediatria.

Antunes, J.L.F., \& Narvai, P.C., (2010) políticas de saúde bucal no brasil e seu impacto sobre as desigualdades em saúde. Rev Saúde Pública. 44:360-5.

Batista, Moura, T.R., Vasconcelos, Gadelha, M. \& Vasconcelos,Gadelha, R. (2020) Fisiopatologia da cárie dentária: Entendendo o processo carioso. Salusvita, Bauru. v. 39, n. 1, p. 169-187.

BRASIL (2020). Agência Nacional de VIgilância Sanitária (ANVISA), nota técnica GVIMS/GGTES/ANVISA NO 07/2020. Brasília.

Bueno, A., Diniz, C., Gonçalves, M., \& Penido, C. (2020) Proposta de isolamento para aplicação de diamino fluoreto de prata. v. 30 , n. 1-2.

Francelino, V., Seixas, G., Neto, P., Yokoyama, M., Porcelli, I., \& Maciel, S. (2019) Eficácia do diamino fluoreto de prata aplicado em diferentes concentrações: Revisão de literatura. Revista Uningá, v. 56, n. S5, p. 12-22. ISSN 2318-0579.

Coelho, C. S., Fedechen, M. C., Volpini, R. C., Pedron, I. G., Kubo, H., Friggi, M. N. P., \& Shitsuka, C. (2020). Evolução da técnica odontológica do tratamento restaurador atraumático. Research, Society and Development, 9(3), e74932439. https://doi.org/10.33448/rsd-v9i3.2439

Costa, I. C. P, Sampaio, R. S, Souza, F. A. C., Dias, T. K. C, Costa, B. H. S, \& Chaves, E. C. L.(2020) produção científica em periódicos online sobre o novo coronavírus (covid-19): pesquisa bibliométrica.

Cruz ,R., Borges-Andrade ,J., Moscon, D., Esteves, G., Delben, P., Queiroga, F., \& Carlotto, P. (2020) COVID-19: emergência e impactos na saúde e no trabalho.Rev. Psicol., Organ. Trab. vol.20 no.2 Brasília abr./jun. 
Dias, F., Peixoto, Y., Lopes, M., Guiraldo, R., \& Berger, S.(2021) O Uso do Diamino Fluoreto de Prata na Prevenção e Paralisação da Cárie Radicular: uma Abordagem não Invasiva e Eficiente no controle da Doença em Idosos.

Delmaschi, R. C., Veloso, L. C, Santos, N. M., Silva, P .D., Oliveira, B., \& Caldarelli, P. (2018) Uso de diamino fluoreto de prata em crianças atendidas em uma unidade básica de saúde do norte do Paraná. Revista de Saúde Pública do Paraná.

Doremalen, V., Morris, N., Holbrook, D., \& Myndi (2020) Aerosol and Surface Stability of SARS-CoV-2 as Compared with SARS-CoV1.NEJM

Estrela, C. (2018). Metodologia Científica: Ciência, Ensino, Pesquisa. Editora Artes Médicas.

Frias, A. C, Antunes, J. L. F., Junqueira, S. R., \& Narvai „P. C.(2007) Determinantes individuais e contextuais da prevalência de cárie dentária não tratada no Brasil. Rev Panam Salud Frias;(22)4:279-85.

Frencken, J. E., Leal, S. C., \& Navarro, M. F. (2012) Twenty-five-year atraumatic restorative treatment (ART) approach: a comprehensive over view. Clin Oral Invest;16(5), 1337-1346

Garbin, C. A. S., Sundfeld, R. H., Santos K. T., \& Cardoso J. D. (2008) Aspectos atuais do tratamento restaurador atraumático. RFO. 13 (1), $25-29$

Guedes-Pinto, A. C., \& Issáo M.(1999) Manual de Odontopediatria. 10a ed. São Paulo: Pancast. p. 184-5.

Guinancio, J. C., Sousa, J. G. M. de, Carvalho, B. L. de, Souza, A. B. T. de, Franco, A. de A., Floriano, A. de A., \& Ribeiro, W. A. (2020). COVID - 19: Desafios do cotidiano e estratégias de enfrentamento frente ao isolamento social. Research, Society and Development, $9(8)$, e259985474. https://doi.org/10.33448/rsd-v9i8.5474

Kikwilu, E. N., Frencken ,J. E., Mulder, J., \& Masalu, J .R. (2009) Dental practitionerns' attitudes, subjective norms and intentions to pratice Atraumatic Restorative Treatment (ART) in Tanazia. J Appl Oral Sci. 17(2):97-102.

Kutsch, V.K. (2014) Dental caries: an updated medical model of risk assessment. J Prosthet Dent.111(4):280-5. 10.1016/j.prosdent.2013.07.014.

Lana, R. (2020) Emergência do novo coronavírus (SARS-CoV-2) e o papel de uma vigilância nacional em saúde oportuna e efetiva. Cadernos de Saúde Pública, [on-line], v. 36, n.3, 2020 ISSN 1678-4464.

Leal, S. C., Navarro, M. F. L., \& Frencken, J. E. (2012) Potencialização do Tratamento Restaurador Atraumático. Pro-Odonto Prevenção. 5:103-139.

Lima, J. (2007) Dental caries: a new concept. Rev. Dent. Press Ortodon. Ortop. Facial 12 (6).

Maltarollo, T. H., Pedron, I. G., Medeiros, J. M. F., Kubo, H., Martins, J. L., \& Shitsuka, C. (2020). A erosão dentária é um problema! Research, Society and Development, 9(3), e168932723. https://doi.org/10.33448/rsd-v9i3.2723

Massara, M. L. A., Imparato, J. C. P., Wambier, D. S., Noronha, J. C., Raggio, D. P., \& Bonecker, M. (2021) Tratamento Restaurador Atraumático Modificado (ARTm). Pesq Bras Odontopediatria.

Massara, M. L. A., Wambier, D. S., \& Imparato, J. C. P. (2010) Tratamento restaurador atraumático. In: Massara MLA, Rédua PCB (coordenadores). Manual de Referência para Procedimentos Clínicos em Odontopediatria. São Paulo: Santos. p. 156-163.

Meng, L., Hua, F., \& Bian, Z. (2020) Coronavirus Disease 2019 (COVID-19): Emerging and future challenges for dental and oral medicine. J Dent Res, 99, 481-7.

Monnerat, A .F., Souza, M. I., \& Monnerat A. B. L. (2013) Tratamento Restaurador Atraumático. Uma técnica que podemos confiar? Rev. bras. Odontol.70 (1), 33-6.

Moraes, Érica B. de, Sanchez, M. C. O., Valente, G. S. C., Souza, D. F. de, \& Nassar, P. R. B. (2020). A segurança dos profissionais de saúde em tempos de COVID-19: uma reflexão. Research, Society and Development, 9(7), e134973832. https://doi.org/10.33448/rsd-v9i7.3832

Munhoz, R. P. (2020) Neurological complications in patients with SARS-CoV-2 infection: a systematic review. Arquivos de Neuropsiquiatria. v. 78, n. 5, p. 290-300.

Navarro, M. F., Leal, S. C., Molina, G. F., \& Villena, R. S. (2015) Tratamento Restaurador Atraumático: atualidades e perspectivas. Rev Assoc Paul Cir Dent. 69 (3), 289-301.

Organização Mundial Da Saúde. - OMS (2020) Doença por coronavírus (COVID-19)

Organização Pan-Americana Da Saúde -OPAS. (2020). Folha informativa sobre COVID-19

Pinheiro, C. F., Melo, M. P. F., Silva, R. R. da, Pedron, I. G., \& Shitsuka, C. (2021). Lesões não cariosas: revisão de literatura. E-Acadêmica, 2 (2), e042227. https://doi.org/10.52076/eacad-v2i2.27

Pinto, L. G., Oliveira, J. J. M. de, Andrade, K. da S., Farias, M. F., Figueiredo, N. F. D. de, Romão, T. C. M., \& Costa, D. F. N. (2020). Recomendações de práticas odontológicas diante à pandemia de Covid-19. Research, Society and Development, 9(7), e634974569. https://doi.org/10.33448/rsd-v9i7.4569

Risemberg, R. I. S., Silva, A. K. R. O. da, Pedron, I. G., Shitsuka, C., \& Maltarollo, T. H. (2021). Conhecimento dos responsáveis sobre o dentifrício fluoretado e fluorose. E-Acadêmica, 2(2), e022226. https://doi.org/10.52076/eacad-v2i2.26

Rocha S. S. D., Joye C. R., \& Moreira M M. (2020) A Educação a Distância na era digital: tipologia, variações, uso e possibilidades da educação online. RSD [Internet]. $9^{\circ}$ de abril de 2020 [citado $4^{\circ}$ de setembro de 2021];9(6):e10963390. Disponível em: https://rsdjournal.org/index.php/rsd/article/view/3390 
Rodrigues, M. (2019) O uso do diamino fluoreto de prata: revisão narrativa atual. Escola Superior de Saúde Fernando Pessoa.

Santana, I. V. G., Valeretto, D. D. B. Cruz, M. C. C., Fernandes, K. G. C., \& Sakashita, M. S. (2018). P o52 - Evolução científica sobre cárie dentária: revisão de literatura.

Santo E do E. (2016) Ensinar e aprender na Educação a Distância: um estudo exploratório na perspectiva das práticas tutoriais. RSD [Internet]. $8^{\circ}$ de dezembro de 2016 [citado $4^{\circ}$ de setembro de 2021];3(2):92-114. Disponível em: https://rsdjournal.org/index.php/rsd/article/view/16

Santos Junior, V.E., Souza, P.R., \& Rosenblatt, A. (2012) Um recurso para paralisar e prevenir cárie em crianças: Diamino Fluoreto de Prata. RFO UPF 17 (2): 228-33.

Shitsuka, C., Friggi, M. N. P., \& Volpini, R. M. C. (2019). Influência dos pais sobre o comportamento infantil no atendimento odontológico. Research, Society and Development, $8(7)$, e43871154. https://doi.org/10.33448/rsd-v8i7.1154

Yuen, Kit-San; Ye, Zi-Wei; \& Fung, Chin-Yee (2020) SARS-CoV-2 and COVID-19: The most important research questions.Cell \& Biosciens

Sousa, E., Crescente, C. Yoshikawa, A., \& Santos, M.(2021) O diamino fluoreto de prata no controle da cárie na primeira infância durante a pandemia da COVID- 19. Research, Society and Development. v. 10, n. 6, e7710615380.

Valente G S C, Moraes Érica B de, Sanchez M C O, Souza D F de, \& Pacheco M C M D. (2020) O ensino remoto frente às exigências do contexto de pandemia: Reflexões sobre a prática docente. RSD [Internet]. $9^{\circ}$ de setembro de 2020 [citado $4^{\circ}$ de setembro de 2021 ];9(9):e843998153. https://rsdjournal.org/index.php/rsd/article/view/8153

Wambier, D .S., \& Bosco, V. L. (1995) Uso de cariostático em odontopediatria: diamino fluoreto de prata. Rev Odontopediatria 4(1):35-41

WHO. Report of the WHO-China Joint Mission on Coronavirus Disease 2019 (COVID-19). (2020)16-24 